\title{
The Role of the Middle Management in Different Segments of the Schooling Path
}

\author{
Dr. Katerina Cerma Cycja \\ Department of Psychology, "Aleksandër Moisiu" University, Durrës, Albania \\ Email: keticycja@yahoo.it
}

\section{Doi:10.5901/ajis.2016.v5n2p63}

\begin{abstract}
This research focuses on middle management (so called "funzioni strumentali"), in Italian schools, seen as a transversal role that represent a model of organization anchored to the intermediate roles, that are able to support and drive the complex management of the school system. The school itself is at the same time an independent organization as well as a public service that provides its specific task in order to deliver its educational aim. As results, it is a complex organization composed by many variables and rules, both formal and informal, which create a unique setting of relationship between different professionals who work there. In order to face all the concerns and discussions addressed to the educational system by the organization itself and its users, we should focus on the organization of roles, functions and professionalism that characterizes the school nowadays. Overall, it should be noted that these particular middle managers are qualitative professionals working in schools. In an attempt to clarify the different facets in which are represented, their position seems to slip between staff leadership and extra-disciplinary specializations, leaving a complex interactions system without a clear division of roles and responsibilities. Probably, at an institutional level, the lack of an overall vision of the school system affects the definition of the tasks and responsibilities of these managers, leaving space to interpretation on their actual role. In an attempt to clarify different facets of them, it seems that the role of the instrumental functions differs in various segments of the schooling path.
\end{abstract}

Keywords: School autonomy, middle management, middle managements role, "funzioni strumentali" (instrumental functions).

\section{Introduzione}

II sistema italiano d'istruzione e formazione ha conosciuto profondi cambiamenti sul piano educativo e culturale. La trasformazione è seguita dell'aumento di valore non solo delle scuole, ma anche di loro personale professionale. II comportamento organizzativo si adegua alla logica dei sistemi "loosely coupled" (Weick, 1976); (S; Zan, 1988) con legami comunitari (Sergiovanni, 2000) sono autonome e inseguono un progetto culturale ed educativo (Schein, 1985). ॥ modello di adhocrazia professionale (Mintzberg,1983) e del professionista indipendente (Orsi, 2002) è espressione di collaborazione al lavoro e della gestione e condivisione delle competenze di leadership organizzativo e didattico (Benadusi \& Serpieri,2000) e di management, intesa come impresa della comunità ed espressione primaria della sua identità (Paparella, 2009, p.115).

Negli ultimi anni la dimensione organizzativa delle istituzioni scolastiche è cresciuta, contemporaneamente avverte la necessità di modificare alcuni componenti importanti del assetto organizzativo sul quale è stato retto tradizionalmente e che le riforme non hanno seguito adeguatamente secondo le necessità specifiche. Non è difficile notare situazioni incoerenti e poche chiare da parte normativa, a seguito dei decreti delegati del '74 del secolo scorso. L'autonomia funzionale delle scuole (legge 59/97 art.21), che s'inserisce nell'ambito della definizione dei rapporti tra le istituzioni, servizi pubblici e cittadini, converge nello stesso assetto organizzativo di quello precedente. Oltre ad avere riflessi sui rapporti esterni all'istituzione scolastica, l'autonomia funzionale è mancata nell'avere riflessioni all'interno delle singole strutture e la ridefinizione della figura del docente.

Dal punto di vista organizzativo, la scuola ha una struttura molto piatta, con un nucleo operativo di insegnanti dotati di un'adeguata preparazione delle discipline diverse e il vertice che è dirigente scolastico. Con l'introduzione dell'autonomia, si è sentita l'esigenza di affiancare al dirigente scolastico, rimasto solo di fronte alla complessità, i due collaboratori da lui individuati ${ }^{1}$, chiamati a operare secondo un rapporto fiduciario e l'altro che si occupa di servizi generali amministrativi (DSGA), con un ruolo strategico all'interno dell'istituto.

1 "Nello svolgimento delle proprie funzioni organizzative amministrative il dirigente può avvalersi di docenti da lui individuati, ai quali possono essere delegati specifici compiti”, D.Lgs. 165/2001, art.25, comma 5. 
La struttura organizzativa esistente non prevede livelli intermedi (middle management) tra la dirigenza e i docenti. I middle management (le funzioni strumentali) sono previste solo dal Contratto di Lavoro. II contesto organizzativo ha favorito il processo di nascita di middle management. La pluralità e varietà della domanda ha bocciato il modello della scuola sull'offerta formativa unica ed eguale per tutti, ormai inadeguata e anacronistica, in conseguenza di ciò si è avvertito il bisogno di trasformarsi in una struttura complessa, sede di un incrocio di competenze fra loro differenti e complementari (Rembado, 2011). Con le innovazioni introdotte dall'autonomia, la scuola ha lasciato la logica dell'obbedienza e ha avviato un processo di corresponsabilità che comporta la capacità di interpretare le esigenze del territorio, coniugandole con l'appartenenza al sistema. Per affermare un'identità peculiare che si traduce nel POF, il quale diventa un banco di prova di maturità professionale, occorre coinvolgere tutti i soggetti operandi nell'istituzione scolastica, i quali da erogatori di sapere divengono protagonisti di un'azione collettiva. Tali attività sono coordinate dal dirigente della scuola, ma, di fatto, svolte da un certo numero di docenti che senza abbandonare la propria attività originaria svolgono anche altri compiti all'interno della scuola, come espressione di una strategia di supporto organizzativo per la scuola.

II quadro istituzionale governativo è stato incerto con il timore di costruire una struttura di staff con competenze e profilo definito e un possibile sviluppo professionale. Per evitare ogni rischio, nel 2002 si è arrivati alla versione debole dello staff, costituito dalle attuali funzioni strumentali2 (Drago, 2007, p.97), che nel tempo hanno svuotato il potenziale della differenzazione e valorizzazione dei profili professionali interni (Previtali, 2012). Le funzioni intermedie della scuola non trovano ancora un'opinione ampiamente condivisa. Per diversi studi: (Cerini, 2002) (Drago, 2007) (Summa, 2012), (Domenici \& Moreti, 2011), tutti i tentativi di articolare, specializzare e differenziare il ruolo delle funzioni strumentali per affrontare le sfide provenienti dall'autonomia, sono stati continuamente rivisti e ridefiniti fino a diventare confusi.

\section{Studio Esplorativo ${ }^{3}$}

L'indagine ${ }^{4}$ è stata eseguita in 39 scuole (plessi) di tutti i cicli d'istruzione: 20 (tra licei, istituti tecnici e istituti professionali), 7 scuole secondarie di I grado, 12 scuole primarie, della provincia di Bari e Taranto. La ricerca si è calata nell'ambito organizzativo interno della scuola coinvolgendo direttamente gli insegnanti delle funzioni intermedi. Per lo strumento d'indagine costruito ad hoc, sono stati presi in considerazione alcuni aspetti del ruolo e alcuni aspetti di job description (Slocum \& Hellriegel, 2010. p.7-8) elaborando l'insieme un'intelaiatura di base. La verifica dello strumento è seguita trame uno Studio Pilota in via preliminare e sperimentale a diversi insegnanti di L. C. Socrate di Bari e L. C. Casardi di Barletta. Le indagini sono stati svolti con la presenza del'intervistatore. Per tutte l'elaborazione dei dati e le operazioni è stato utilizzato il software SPSS.

I risultati confermano che i middle management possiedono requisiti e abilità specifiche come capacità di elaborare programmi operativi e la stesura specifica e formalizzata di progetti di lavoro. Le loro competenze si sono costruite mediante lo svolgimento dell'incarico come Experiential Learning (Kolb, 1984) che delinea il percorso di apprendimento come formazione continua. Dai risultati emerge che il comportamento organizzativo degli istituti scolastici si sta orientando verso il superamento del principio della rotazione. L'88,1\% risultano aver ricoperto lo stesso incarico in precedenza e le loro competenze possono essere descritte in termine di saper agire. La presenza delle reti di risorse organizzative esprime il loro poter agire Sorprendente è la conferma che l'87,4\% risultano prevalentemente autocandidature. Quindi a loro non basta il know how, specifica del docente, ma contano di più sulle loro potenzialità e lo manifestano per far progredire la vera e propria professionalità. II loro voler fare, completa il ciclo secondo il concetto di Le Boterf (Le Boterf, 1997) in termine di performance professionale. La formazione di middle management non è prevista in nessun modo, quindi le loro competenze sono il risultato di un approccio formativo trame le tecniche OJT (On- theJob-Training).

Le "funzioni strumentali" agiscono con competenza per stabilire contatti con diversi interlocutori, e gestire i progetti formativi con Enti pubblici o privati e in generale con il territorio, la quale non fa parte nelle competenze comuni dell'insegnante. In questo caso si tratta di una competenza differenziata (Lordit, 2011), una competenza convergente che diventa un ulteriore specializzazione di carattere funzionale ed organizzativo di natura orizzontale, quindi una

\footnotetext{
2art. 33.CCNL. 2007

${ }^{3}$ Cerma Cycja, K. Dottorato di ricerca (in Psicologia del Lavoro e delle Organizzazioni, (2015) : Il presidio operativo dei quadri intermedi "anello mancante" della struttura organizzativa interna alla scuola. L'indagine empirica è stata condotta nel periodo compreso tra settembre 2013 e marzo 2014.

4 Una bozza del progetto di ricerca è stata presentata all'USR di Puglia (Ambito territoriale per la provincia di Bari e Ambito territoriale per la Provincia di Taranto) che l'ha apprezzato e si è incaricata di concordare con delle scuole per proseguire con l'indagine.
} 
differenziazione funzionale su aree non disciplinari.

Gli aspetti emersi offrono altri spunti interessanti di riflessione verso l'identificazione del ruolo delle funzioni strumentali, il quale risulta variato rispetto ai diversi segmenti del percorso di istruzione scolastica Trame le differenze statisticamente significative fra le tipologie di scuole, nella media delle risposte in una serie di componenti di rilevante importanza emergono delle configurazioni di funzioni intermedi di diverso spessore.

II corrispettivo della prestazione, determinato sulla base delle caratteristiche e della consistenza delle'attività svolte, formula la graduatoria secondo l'ordine decrescente partendo dalla scuola secondaria di secondo grado nella prima posizione, seguito dalla secondaria di primo grado e all'ultima posizione la scuola primaria. (Grafico 1)

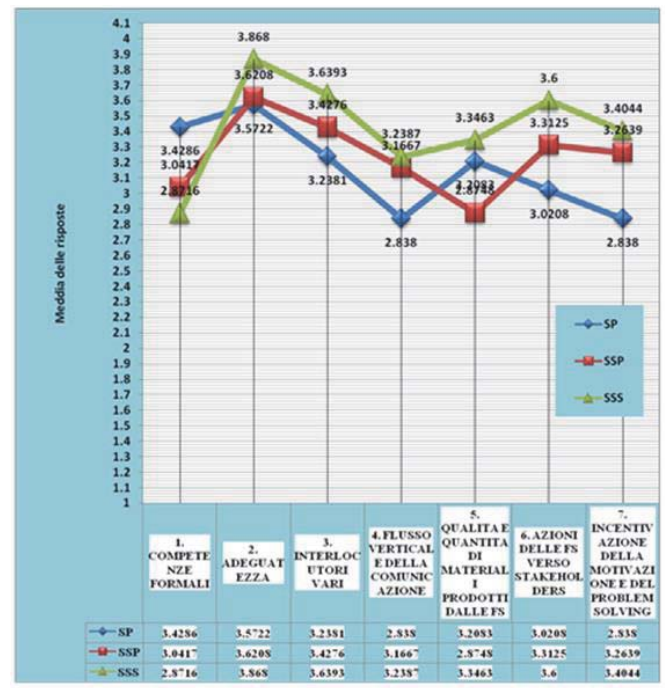

Grafico 1. Differenze statisticamente significative fra le tipologie di scuole nella media delle risposte in una serie di componenti (SP- scuola primaria, SSP- scola secondaria di primo grado, SSS-scuola secondaria di secondo grado).

I - emerge una differenza nella percezione del possesso dei requisiti delle funzioni strumentali fra S scuola primaria e secondaria di secondo grado in favore della prima. Tale differenza risulta coerente con la percezione delle funzioni strumentali, da parte della scuola primaria quando ultimamente, a partire dall'anno accademico 2011/2012, per intraprendere la professione di insegnante di scuola primaria è necessario conseguire la laurea magistrale a ciclo unico, di durata quinquennale in scienze della formazione primaria (classe LM 85 bis). Inoltre si necessitano percorsi di specializzazione per l'acquisizione delle competenze linguistiche dell'inglese, secondo il quadro europeo di riferimento, e lo sviluppo delle competenze digitali e didattiche. Dai nostri dati risulta che $11,1 \%$ di partecipanti delle funzioni strumentali nelle scuole primarie è in possesso solo di diploma della scuola superiore di secondo grado.

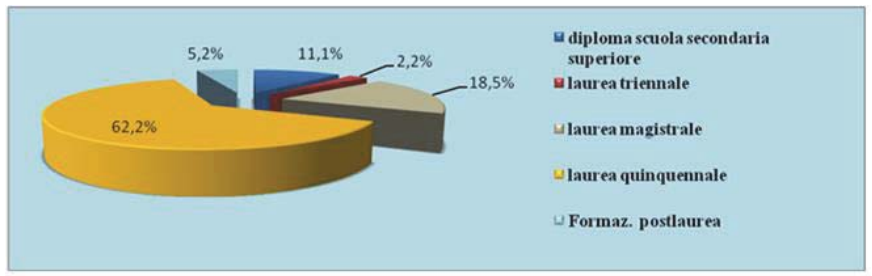

Grafico 2. Titolo di studio

II - emerge una differenza nella percezione delle funzioni strumentali, come strumento adeguato per migliorare l'efficienza organizzativa fra scuola secondaria di secondo grado e scuola primaria in favore della prima. Tale differenza 
probabilmente risulta coerente rispetto all'organizzazione dell'istruzione scolastica, secondo i segmenti di un itinerario scolastico che abbraccia tre tipologie di scuola, caratterizzate ciascuna da una specifica identità educativa e professionale.

II curricolo degli istituti compressivi è costituito nella logica della verticalità degli interventi educativi e formativi tra segmenti della scuola dell'infanzia, della scuola primaria e della scuola secondaria di primo e secondo grado. La scuola accoglie, promuove e arricchisce l'esperienza vissuta dei bambini in evoluzione. Attraverso il curricolo dell'istituto, i docenti individuano le esperienze di apprendimento più efficaci, le scelte didattiche più efficaci, le strategie specifiche, con l'intensione all'integrazione e la possibile aggregazione fra le discipline. La scuola dell'infanzia come un'agenzia educativa dialoga e collabora con le altre istituzioni per effettuare in modo concreto un'autentica centralità del bambino $\mathrm{e}$ offre un ambiente educativo capace di concedere possibili risposte al bisogno di attenzione e apprendimento. La scuola dell'infanzia realizza un curricolo che rende concrete tutte le dimensioni di apprendimento: sensoriale - corporea, artistica - espressiva, intellettuale, sociale e di cittadinanza 5 .

La scuola primaria mira all'acquisizione degli apprendimenti di base, ai bambini, offre opportunità di sviluppare le dimensioni cognitive, emotive, affettive, sociali, corporee, etiche e di acquisire i saperi irrinunciabili. Si colloca come scuola formativa che, attraverso di ciascuna disciplina permette di esercitare differenti stili cognitivi (Moncada, 2016), ponendo le premesse per lo sviluppo del pensiero riflessivo e critico.

Per quanto riguarda la scuola secondaria di secondo grado i fini organizzativi e della gestione sia dal punto di vista metodologico - didattico nell'ambito di un processo educativo vede come protagonisti soggetti nella fase più importante della loro formazione culturale e professionale.

Probabilmente nelle scuole secondarie di secondo grado, come tipologia di scuola con le sue specifiche identità educative e professionali, le funzioni strumentali sono percepite come degli strumenti adeguati ed efficienti dal punto di vista organizzativi.

Nelle scuole d'infanzia come segmenti iniziali d'itinerari scolastici l'attività scolastica si svolge da due docenti, e quelle primarie da tre docenti tra qui uno di loro svolge l'attività di tutoraggio come docente prevalente. Il docente tutor è il docente al quale è richiesto il possesso di specifica formazione e la capacità di instaurare un costante rapporto con le famiglie e con il territorio, al fine di svolgere, avvalendosi del contributo degli altri insegnanti, compiti di orientamento, riguardo alla scelta delle attività e degli insegnamenti rientrati nell'offerta facoltativa organizzata dalle scuole. Svolge compiti di coordinamento delle attività educative e didattiche, cura delle relazioni con le famiglie e di documentazione del percorso formativo compiuto dall'alunno. Quindi, il tutor scolastico rappresenta una risorsa contrapposta più che complementare alla figura docente, che può essere letta in molti modi. Probabilmente una parte dell'insieme delle attività di funzioni strumentali si svolge in qualche modo dai docenti tutor, rendendo le prime meno visibili, ma anche forse meno adeguate e meno efficienti come parte organizzativa interna.

III - emerge una differenza nella percezione dei rapporti che stabiliscono le funzioni strumentali con vari interlocutori, fra la scuola secondaria di secondo grado e scuola primaria, in favore della prima. Probabilmente la differenza emerge dalla diversità degli obiettivi che compongono l'orientamento per ciascun segmento del percorso formativo. La scuola secondaria di secondo grado di solido articola la sua azione di orientamento in uscita, con precise finalità come: coinvolgere gli studenti degli ultimi anni in attività che aiutino a riflettere sul proprio percorso formativo, spingere a operare a loro una scelta consapevole sul proseguimento degli studi o su un percorso formativo professionalizzato, fornire consulenza, supporto e monitoraggio delle attività medesime. Per la realizzazione di tali finalità gli istituti scolastici costruiscono i propri interventi al perseguimento degli obiettivi come: far riflettere gli studenti sui propri interessi e sul proprio percorso formativo, far emergere e valorizzare le attitudini personali, vagliare le proprie competenze ecc. Invece la scuola primaria prosegue con degli obiettivi come: rivelazione del livello di formazione in itinere, programmazione di progetti di continuità formativa, certificazione delle competenze disciplinari, programmazione di percorsi d'inserimento per $\mathrm{BES}^{6}$, programmazione di azioni di ponte tra i livelli di istruzione ecc.

IV - emerge una differenza nella percezione del flusso della comunicazione e informazione esercitato dalle funzioni strumentali fra la scuola secondaria di secondo grado e la scuola primaria a favore della prima. E' evidente che le differenze nascono dai livelli diversi del percorso di istruzione e formazione.

II progetto culturale ed educativo della scuola primaria, pretende di essere svolto secondo un passaggio continuo

${ }^{5}$ Atto di indirizzo recante $i$ criteri generali necessari ad armonizzare gli aspetti pedagogici didattici ed organizzativi relativi alla Scuola dell'Infanzia e al I ciclo di istruzione. Ministero dell'Istruzione, dell'Università e della Ricerca (2009)

${ }^{6}$ Bambini con Bisogni Educativi Speciali. 
che va da una impostazione unitaria predisciplinare all'emergere di ambiti disciplinari progressivamente differenti. La programmazione didattica ha un valore importante e disegnerà i percorsi e le procedure più idonee per lo svolgimento dell'insegnamento, tenendo conto che i risultati devono essere simili qualsiasi sia l'itinerario metodologico scelto. Per di più la scuola elementare si articola in due cicli. L'unitarietà dell'insegnamento che rappresenta la caratteristica educativa didattica peculiare della scuola elementare è assicurata dallo specifico dell'insegnante di classe (nel primo ciclo). Probabilmente, nelle scuole primarie il flusso di comunicazione e informazione è steso di più sul piano orizzontale tra $i$ colleghi docenti che sul piano verticale.

Al secondo ciclo si prevede l'utilizzazione di una pluralità di docenti e il modulo base dell'organizzazione didattica deve basarsi sulla valorizzazione delle esperienze e degli specifici interessi culturali degli insegnanti. Evidentemente le funzioni strumentali comunicano di più sul piano verticale con la dirigenza mostrandosi di più in staff.

$V$ - emerge una differenza nella percezione della qualità e quantità dei materiali prodotti dalla scuola secondaria di primo grado e la scuola superiore di secondo grado in favore della seconda. Probabilmente tale differenza emerge dalle tipologie diverse di istituti delle scuole secondarie di secondo grado: licei, istituti tecnici e istituti professionali e l'ampliamento dell'offerta formativa. L'introduzione delle sperimentazioni ha determinato un diffuso rinnovamento dell'offerta didattica che oggi si può rilevare con la numerosità delle tipologie di corsi offerti da ciascuna scuola. Molte scuole oltre alla combinazione del corso di ordinamento e sperimentazione dello stesso indirizzo, prevedono anche corsi di sperimentazione di indirizzo diverso dal principale. ${ }^{\text {. }}$

$\mathrm{VI}$ - emerge una differenza nella percezione delle azioni di funzioni strumentali verso gli stakeholder tra la scuola secondaria di secondo grado e la scuola primaria in favore della prima. Tale differenza probabilmente è legata dagli obiettivi di due livelli di istruzione distanti uno con l'altro. I principali stakeholder, identificati solitamente da tutti gli istituti scolastici indipendentemente dal livello della scuola, di solido sono: interni (come studenti, le famiglie, docenti, il personale non docente, le scuole in rete, il comune e il quartiere, la provincia, la Regione, Ufficio Scolastico Regione) e gli esterni (come le università, diverse associazioni senza finalità di lucro, l'azienda USL per il PEI). Le scuole secondarie di secondo grado, soprattutto quelle di indirizzo tecnico o professionale con il piano d'orientamento in uscita necessitano di altri stakeholder come le università, ma anche aziende e istituzioni per agevolare e sostenere l'ingresso dei giovani nel mondo di lavoro, contatti con delle Associazioni giovani industriali o la Camera di Commercio per inserimento CV dei diplomati su banca dati, inserimento sui progetti di imprenditorialità giovanile, convenzioni e protocolli d'intesa e accordi con enti, istituzioni e aziende ecc.

VII - emerge una differenza nella percezione della definizione del ruolo operativo delle funzioni strumentali verso l'incentivazione della motivazione e del problem solving, tra la scuola secondaria di secondo grado e la scuola primaria a favore della prima. La differenza probabilmente si spiega con rispettivi progetti distinti delle funzioni strumentali secondo l'ordine di scuola II ritorno del maestro unico nelle primarie costituisce un punto di riferimento forte, sia sul piano didattico che su quello affettivo - relazionale. Ciò che diventa importante è che l'azione educativa è costruita con lui e intorno a lui, quindi il progetto unitario è inspirato da una visione unitaria del sapere di natura predisciplinare, strettamente correlata alla realtà e all'esperienza di vita dei bambini. È evidente che l'incentivazione della motivazione e del problem solving verso i bambini è più affidata al suo punto di riferimento, che in questo caso è il maestro unico in rapporto con qualsiasi altro insegnante con incarico delle funzioni strumentali a prescindere dalla sua formazione e livello specialistico. Nella scuola secondaria di secondo grado le funzioni strumentali si organizzano in una modalità più articolata e generalmente con precisi obiettivi. Si mettono in campo diverse strategie di supporto per l'orientamento come l'attivazione dello sportello d'orientamento dotato di front office che mira erogazione di informazioni e back office per la raccolta, la catalogazione e sistemazione dei strumenti informativi. Non di raro le funzioni strumentali attivano azioni di accompagnamento e tutorato, con gli obiettivi del counseling scolastico, che convergono verso la prevenzione del disagio attraverso il sostegno e l'ascolto attivo, la responsabilizzazione in collaborazione con il psicologo e mirano ad aumentare la motivazione scolastica e stimolare l'apprendimento ma anche facilitare i rapporti studenti-docenti.

\section{Conclusioni}

Nella scuola secondaria di secondo grado, le funzioni strumentali emergono all'interno dell'organizzazione scolastica come un elemento organizzativo interno più stabile, privo d'investitura formale ma liberamente articolabili negli specifici

${ }^{7}$ I percorsi formativi della scuola secondaria di secondo grado statale tra corsi di ordinamento, sperimentazioni e autonomia, in http://archivio.pubblica.istruzione.it/news 
settori della complessità dell'organizzazione scolastica che operano come centro di consulenza e di supporto attraverso le azioni di empowerment in un ottica di responsabilizzazione e valorizzazione delle risorse professionali. Si profilano come strumento adeguato per aumentare l'efficienza organizzativa, instaurano e coordinano rapporti con vari interlocutori, raccolgono le informazioni di feedback e comunicano con il vertice con un flusso verticale di informazioni. Stabiliscono rapporti di qualità con diversi stakeholder con i quali interagiscono 0 entrano in contatto con l'obiettivo di identificare e comprendere i bisogni e le aspettative di diverse categorie. Inoltre svolgono un ruolo sempre più importante verso l'incentivazione della motivazione e del problem solving.

Nelle scuole secondarie di primo grado e ancora di più in quelle primarie probabilmente le funzioni strumentali stanno ancora in fase di consolidamento del ruolo.

\section{References}

Baldassarre, S., Bonani, G. P. (2004). Autoformazione. Come liberarsi dagli stereotipi formativi d'impresa. Milano: Franco Angeli.

Benadusi, L., Serpieri, R. (2000a) (a cura di), Organizzare la scuola dell'autonomia, Roma, Carocci.

Cerini, A. (2002). Una nuova condizione professionale per gli insegnanti, in Drago, A. Professionalità docente e codice deontologico degli insegnanti, (pp. 29-30). Trento: Erickson.

Domenici, G., Moreti, G. (2011).Leadership educativa e autonomia scolastica: il governo dei processi formativi e gestionali nella scuola di oggi. Armando Editore.

Drago, R. (2007). Leadership educativa e middle management. in http://www.treellle.org/files/ll/seminario089.pdf. TREELLE Le ricerche n. $x-0729-10-2007$. [consultato il 03/05/2013]

Kolb, D. A. (1984).Experiental Learning experience as the source of Learning and Cevelopment. Englewood Cliffs :NJ, Prentice Hall.

Le Boterf, G. (1997). De la compètence à la navication profetionelle. Paris: Edition d'organisation.

Lordit, L. (2011). Modelli di reclutamento, formazione, sviluppo e valutazione degli insegnanti. Trento: Iprase.

Mintzberg, H. (1983). Strutture in Fives: Designing Effetive Organizations, Prentice-Hall, Englewood Cliffs (trad.it. La progettazione dell'organizzazione aziendale, II Mulino, Bologna, 1985).

Moncada, S (2016). II concorso a cattedre nella scuola primaria e secondaria di primo grado. Manuale per la prova scritta e orale, (pp. 36), Maggioli Editore

Orsi M., (2002). Scuola, organizzazione, comunità, Brescia: Editrice La Scuola.

Paparella, N. (2009). Piano dell'Offerta Formativa. in Paparella, N. (a cura di), II progetto educativo (Volume Secondo). Comunità educante, opzioni, curriculi e piani; ( pp. 178-187), Roma: Armando.

Previtali, D. (2012) Figure di staff in Europa, Rivista dell'istruzione, 4, (pp. 46-48).

Rembado, G. (2011). Middle management nelle scuole, Notiziario della struttura regionale del Piemonte, (2), (10 gennaio 2011), (pp.16).

Schein, E.H. (1985). Organisational Culture and Leadership. Jossey Bass, San Francisco.

Sergiovanni, T. J. (2000). Costruire comunità nella scuola. Roma: Editrice LAS.

Slocum, J. W., Hellriegel, D. Jr. (2010).Comportamento organizativo. fondamenti psicologici per mangeriale. Ulrico Hoepli Editore, Milano

Summa, I. (2012). II middle management: cos'è e a cosa serve. Rivista dell'istruzione, 4, (pp. 4-9).

Weick, K. E. (1976). Educational organizations as loosely coupled system, in Amministrative Science Quartely, 21. (pp.1-19). (trad. it. Le organizzazioni scolastiche come sistemi a legame debole. In Zan, S. (a cura di) Logiche d'azione organizzativa (pp. 355-379). Bologna, II Mulino, 1988.

Zan, S. (1988). (a cura di), Logiche di azione organizzativa, Bologna: II Mulino. 